\title{
Evaluación fenotípica de semillas de haba (Vicia faba L.) colectadas en Nariño-Colombia
}

\section{Phenotypic evaluation of broad bean (Vicia faba L.) seeds collected in Nariño-Colombia}

\author{
David Álvarez-Sánchez ${ }^{1}$; Claudia Salazar-González²; Benjamín Sañudo-Sotelo ${ }^{3}$; Carlos Betancourth-García ${ }^{4}$ \\ 1'Ing. Agrónomo, M.Sc., Ph.D. Universidad de Nariño, Facultad de Ciencias Agrícolas. Pasto - Nariño, Colombia; e-mail: daealvarezsa@udenar.edu.co; iD https:// \\ orcid.org/0000-0003-3563-2529 \\ 2Ing. Agrónomo, M.Sc., Ph.D. Universidad de Nariño, Facultad de Ciencias Agrícolas. Pasto - Nariño, Colombia; e-mail: claudiasalazarg@udenar.edu.co; iD https:// \\ orcid.org/0000-0002-5461-2761 \\ ${ }^{3}$ Ing. Agrónomo. Universidad de Nariño, Facultad de Ciencias Agrícolas. Pasto - Nariño, Colombia; e-mail: benjaminsañudo@yahoo.es; iDhttps://orcid.org/0000- \\ 0001-9943-5650 \\ ${ }^{4}$ Ing. Agrónomo, M.Sc., Ph.D. Universidad de Nariño, Facultad de Ciencias Agrícolas. Pasto - Nariño, Colombia; e-mail: cbet70@udenar.edu.co; iDhttps://orcid. \\ org/0000-0001-6573-4230
}

Cómo citar: Álvarez-Sánchez, D.; Salazar-González, C.; Sañudo-Sotelo, B.; Betancourth-García, C. 2021. Evaluación fenotípica de semillas de haba (Vicia faba L.) colectadas en Nariño-Colombia. Rev. U.D.C.A Act. \& Div. Cient. 24(2):e1874. http://doi.org/10.31910/rudca.v24. n2.2021.1874

Artículo de acceso abierto publicado por Revista U.D.C.A Actualidad \& Divulgación Científica, bajo una Licencia Creative Commons CC BY-NC 4.0

Publicación oficial de la Universidad de Ciencias Aplicadas y Ambientales U.D.C.A, Institución de Educación Superior Acreditada de Alta Calidad por el Ministerio de Educación Nacional.

Recibido: febrero 21 de 2021

Aceptado: octubre 25 de 2021

Editado por: Helber Adrián Arévalo Maldonado

\section{RESUMEN}

El haba es una leguminosa asociada a sistemas campesinos de clima frío. En Colombia, se cultiva, principalmente, en el departamento de Nariño, donde se ha visto reducida el área y el número de materiales sembrados, debido a la disminución en los niveles de rendimiento del cultivo y la agresividad de los problemas fitosanitarios. Este estudio evaluó la diversidad de esta leguminosa, a partir de la forma y color de la semilla, en una colección de 60 accesiones de haba, discriminadas en siete variedades. Mediante el procesamiento de imágenes digitales y medición manual, se determinó la longitud polar (LP), longitud ecuatorial (LE), relación LP/LE, área (AR), grosor (GR) y peso de cien semillas (P100); además, se calcularon los parámetros de color, basados en la escala CIELab ( $L^{*}, a^{*}$ y b*). Las variables, se analizaron con estadística descriptiva, correlación de Pearson y el método multivariado de componentes principales (ACP). Los resultados identificaron alta diversidad de fenotipos, caracterizando, cuantitativamente, las siete variedades. Se presentó correlación alta entre las variables LP, LE y AR, igualmente, entre $L^{*}$ y b*. Con el biplot de los resultados de ACP, se ratificó la variabilidad de la colección y posibilitó generar una agrupación preliminar. La investigación, se puede tomar en cuenta como base para el desarrollo de trabajos de conservación y de evaluación agronómica de esta leguminosa.

Palabras clave: Color; Leguminosas; Morfometría; Recurso genético; Variación fenotípica.

\section{ABSTRACT}

Fava bean is a legume associated with cold climate farming systems. In Colombia, it is grown mainly in the department of Nariño, 
where the area and number of planted materials have been reduced due to the decrease in crop yield levels and the aggressiveness of phytosanitary problems. This study evaluated the diversity of this legume based on seed shape and color in a collection of 60 bean materials divided into seven varieties. Through digital image processing and manual measurement, polar length (LP), equatorial length (LE), LP/LE ratio, area (AR), thickness (GR) and hundred seed weight (P100) were determined, and color parameters based on the CIELab scale ( $L^{*}, a^{*}$ and $\left.b^{*}\right)$ were calculated. The variables were analyzed with descriptive statistics, Pearson's correlation and the multivariate principal component method (PCA). The results identified a high diversity of phenotypes, quantitatively characterizing the seven varieties. There was a high correlation between the variables LP, LE and AR, as well as between $\mathrm{L}^{*}$ and $\mathrm{b}^{*}$. The biplot of the PCA results confirmed the variability of the collection and made it possible to generate a preliminary grouping. The research can be taken into account as a basis for the development of conservation and agronomic evaluation of this legume.

Keywords: Legumes; Morphometrics; Phenotypic variation; Color; Genetic resource.

\section{INTRODUCCIÓN}

El haba (Vicia faba L.) es una especie subcosmopolita, posiblemente, originada en el Cercano Oriente o en Asia Menor, a partir de ancestros desconocidos (Duc et al. 2015). Pertenece a la familia Fabaceae, que es la más importante del orden Fabales, considerada la tercera familia de las Angiospermas, en cuanto al número de especies y, en el segundo lugar, en importancia económica (De la Rosa \& Fajardo, 2016).

La migración del haba a Sudamérica, especialmente a los Andes, ocurrió durante el siglo $\mathrm{XV}$, junto con la conquista española y portuguesa (Duc et al. 2015), con la introducción de una multiplicidad de materiales, que fueron seleccionados de manera natural, por lo que quedan solo aquellos que se adaptaron a las condiciones de trópico, lo que proporciona una fuente valiosa de variación genética (Horque, 2004).

Las semillas contenidas en vainas, se utilizan, principalmente, para consumo humano secas o frescas; presentan un elevado contenido proteico, que varía entre el 16 y el $35 \%$, de acuerdo con la variedad y las condiciones ambientales (Pérez et al. 2015; Karkanis et al. 2018; Khazaei \& Vandenberg, 2020). Son una fuente de aminoácidos esenciales y poseen altos contenidos de $\mathrm{K}, \mathrm{Ca}, \mathrm{Mg}$, Fe y Zn (Khazaei \& Vandenberg, 2020).

El haba, también se emplea en la alimentación de animales (Duc et al. 2015). Además, por la capacidad que tienen las raíces para establecer simbiosis con las bacterias Rbizobium leguminosarum y con la micorriza vesículo-arbuscular, que le permiten fijar nitrógeno atmosférico, es utilizada para preservar la fertilidad del suelo y generar mayor actividad biológica (Karkanis et al. 2018; Khazaei \& Vandenberg, 2020).
En Colombia, el cultivo se basa en el uso de variedades locales en sistemas campesinos de baja inversión, donde los agricultores destinan la cosecha, especialmente, para autoconsumo. Se registra un promedio de producción de 7.304 toneladas de haba en fresco, en un área sembrada de 1.084 hectáreas, siendo Nariño el principal departamento productor, a nivel nacional, con una concentración del $80 \%$ de la cosecha del país (MADR, 2021).

Cabe señalar que, en las últimas dos décadas, la modificación en la dinámica agrícola de la región altoandina del departamento de Nariño ha causado presión, especialmente, sobre los cultivos de baja rentabilidad económica y de autoconsumo, poniendo en riesgo a diferentes sistemas de producción tradicionales (Álvarez et al. 2019). En el caso del haba, se redujo el área sembrada de 2.000 ha, en 1999, a un estimado de 630 ha, para el 2019, con el consecuente impacto en la disminución de la siembra de diferentes variedades comerciales (Sañudo et al. 1999; MADR, 2021), en la erosión del germoplasma y en la pérdida irreparable de materiales sobresalientes.

A pesar de esto, la variabilidad genética sigue siendo importante en este departamento, especialmente, en los sistemas campesinos, que han contribuido a la protección, la selección e intercambio de materiales de $V$. faba, conscientes del valor que representa esta leguminosa en la dieta y la dinámica agrícola. Practicando por los productores la conservación in situ, en donde se conjugan los saberes del entorno, el comportamiento de la especie y un conocimiento heredado perfeccionado por la experimentación empírica (Díaz et al. 2008).

En este sentido, Herrera et al. (2013) sustentan la importancia de colectar y de estudiar el germoplasma local, dado que esto permite conocer la variación genética e identificar características deseables dentro de la población. Concretamente, en el haba, Duc et al. (2015) y Karkanis et al. (2018) sugieren la evaluación preliminar de los rasgos asociados a las semillas para diseñar, eficientemente, los programas de conservación o selección.

Por lo anterior, el presente trabajo buscó evaluar la variación fenotípica de semillas de haba colectadas en el departamento de Nariño, Colombia. Lo que permitirá tener una determinación aproximada de la variabilidad de $V$. faba, en las zonas donde se cultiva.

\section{MATERIALES Y MÉTODOS}

Colección y disposición de las semillas. Se evaluaron siete variedades de haba de la colección de trabajo del Grupo de Investigación en Sanidad Vegetal GRISAV, obtenidas de lotes fenotípicamente homogéneos y geográficamente aislados, de la región altoandina del departamento de Nariño. El código empleado para identificar las variedades fue: Alp = Alpargata; $\mathrm{Ar}$ $=$ Argentina; $\mathrm{Bn}=$ Beso de novia; $\mathrm{Bc}=$ Blanca común; Ro = Roja; Rs $=$ Rosada y $\mathrm{S}_{\mathrm{v}}=$ Semiverde $($ Tabla 1$)$. 
Tabla 1. Identificación de los materiales de Vicia faba, colectados en el departamento de Nariño, Colombia y depositados en la colección GRISAV.

\begin{tabular}{|c|c|c|c|}
\hline Municipio & $\begin{array}{c}\text { Altitud } \\
\mathbf{m} \text { s.n.m. }\end{array}$ & $\begin{array}{c}\text { Temperatura } \\
\text { promedio } \\
{ }^{\mathbf{o}} \mathbf{C}\end{array}$ & Código \\
\hline Pasto & $2.520-2.870$ & 14,4 & $\begin{array}{c}\text { Bc-1; Bc-2; Ro-3; Bc-4; Alp-5; Bc-10; Bc- } \\
\text { 35; Bc-36; Bc-37; Bc-38; Bc-39; Bc-40; } \\
\text { Ar-41; Bc-42; Bc-43; Bc-44; Bc-45; Bc-46; } \\
\text { Bc-47; Ro-48; Bc-49; Ro-53; Bc-54; Bc- } \\
55 ; \text { Ro-56; Alp-57; Rs-58; Bn-59; Rs-60 }\end{array}$ \\
\hline Ipiales & $2.830-2.980$ & 14,3 & $\begin{array}{c}\text { Ar-15; Ro-17; Sv-26; Ro-27; Ar-28; Ro- } \\
29 ; \mathrm{Bc}-30 ; \mathrm{Ar}-33 ; \mathrm{Bc}-34 ; \mathrm{Bc}-51 ; \mathrm{Bc}-52\end{array}$ \\
\hline Córdoba & $2.760-2.860$ & 13,4 & $\begin{array}{c}\text { Bc-11; Bc-12; Bc-20; Ro-21; Ro-22; Rs- } \\
23 ; \mathrm{Bc}-24 ; \mathrm{Bn}-25\end{array}$ \\
\hline Guachucal & $3.160-3.188$ & 13,3 & Bn-5; Ro-6; Bc-7; Rs-8; Bc-50 \\
\hline Tuquerres & $2.580-3.023$ & 11,1 & Alp-9; Rs-13; Bc-14; Bc-18 \\
\hline Potosí & 2.622 & 12,9 & Rs-19; Bc-31; Ro-32 \\
\hline Carlosama & 2.985 & 17,6 & Bc-16 \\
\hline
\end{tabular}

Cada variedad contó con un número heterogéneo de accesiones Alp (3), Ar (5), Bn (3), Bc (29), Ro (11), Rs (8) y Sv (1), para un total de 60 materiales. En laboratorio, la evaluación morfológica y la estimación del color requirieron de 30 semillas seleccionadas al azar de cada material, así como tres grupos de cien semillas, para determinar el peso.

Captura de imágenes digitales. Se construyó un escenario con luz artificial, porta cámara y una placa de cerámica blanca no refractante, que permitió la captación de imágenes estandarizadas, mediante cámara digital $\left(\mathrm{SONY}^{\circledR}, \mathrm{HX} 300\right)$, dotada con zoom óptico de 50x.

Las fotografías con resolución de $15 \mathrm{MP}$, se procesaron en el software ImageJ 1.53e (National Institutes of Health, Bethesda, USA), teniendo una medida de referencia de tamaño de $10 \mathrm{~cm}$.

Morfología de la semilla. A cada semilla, se le tomaron medidas de longitud en cm. Longitud polar (LP), estimada en el paralelo más alto al hilio y longitud ecuatorial (LE), estimada en la zona media de la semilla; con éstas, se midió la relación longitud polar/longitud ecuatorial (LP/LE); además, en $\mathrm{cm}^{2}$, se calculó el área de la semilla (AR). Los valores obtenidos fueron sometidos sistemáticamente a una comparación manual, usando un calibrador digital (Vinca, DCLA), con una lectura de $\pm 0,01 \mathrm{~cm}$, para comprobar la coincidencia entre los dos métodos.

El grosor (GR), se estimó en la zona media de cada semilla con el calibrador digital y el peso de cien semillas (P100), se determinó en tres repeticiones, utilizando una balanza electrónica, con un error de $0,01 \mathrm{~g}$.

Color de la semilla. Con el uso de las fotografías digitales, se cuantificaron los parámetros de color en el software ImageJ 1.53e, basado en la escala CIELab (International Commission on Illumination), descrita por Yildiz etal. (2016), como: L* (luminosidad) de negro $=0$ a blanco $=100 ;$ cromaticidad $\mathrm{a}^{*}$ de rojo-púrpura $=$ valor positivo a verde-azul $=$ valor negativo $\mathrm{y}$ cromaticidad $\mathrm{b}^{*}$ de amarillo $=$ valor positivo al azul $=$ valor negativo. $\mathrm{Al}$ igual que en la estimación morfológica, se realizó comparación de los resultados proporcionados por el software, con un equipo espectrofotómetro Konica Minolta, CM-5.

Adicionalmente, se identificó el color de hilio $(\mathrm{CH})$, con las categorías: oscuro/claro y color secundario de la semilla (CS) con las categorías: presencia/ ausencia.

Análisis estadístico. Las variables morfológicas y de color de la semilla fueron estudiadas, mediante estadística descriptiva, correlación lineal de Pearson (r) y el método estadístico de componentes principales (ACP). Este último, buscó transformar el conjunto de $p$ variables en componentes independientes, con el fin de estimar la variabilidad existente en la colección y la explorar la posibilidad de generar una agrupación para las 60 accesiones de haba.

Además, a partir de las mediciones realizadas sobre una muestra aleatoria de 100 semillas, se aplicó la prueba de T Student, con un grado de significancia de $\mathrm{p}<0,05$, para contrastar las medias entre el método de estimación manual y digital en las variables LP y LE, así como los parámetros de color $\mathrm{L}, \mathrm{a}^{*}$ y b*, por el método digital y el espectrofotómetro.

Para el análisis de los estadísticos, se utilizó el lenguaje de programación R v.4.1.1 (R Foundation for Statistical Computing, Vienna, Austria), utilizando la función t.test y el paquete Factoextra.

\section{RESULTADOS Y DISCUSIÓN}

Morfología de la semilla. El análisis de imágenes digitales no mostró diferencias significativas respecto a las estimaciones morfológicas realizadas manualmente, como se observa en la tabla 2 , en donde se registró, para las variables LP y LE, un p-valor mayor a $\alpha=0,05$. Al considerar los 1.800 registros de semillas que 
se analizaron, se confirman los beneficios referidos respecto a la confiabilidad y rapidez de la metodología digital, indicados en otras investigaciones (Varga et al. 2019; Di Cecco et al. 2019).
Como resultado, las mediciones resumidas en la tabla 3, sugieren variación entre y dentro de las variedades de haba en la colección. Las primeras variables refieren semillas maduras, con valores promedio en LP, desde 2,28 a 3,05 cm y en LE, desde 1,78 a 2,28 cm.

Tabla 2. Prueba T Student para la igualdad de medias, entre la forma y el color de las semillas de haba, colectadas en el departamento de Nariño, Colombia y depositados en la colección GRISAV $(\mathrm{n}=100)^{*}$.

\begin{tabular}{|c|c|c|c|}
\hline Parámetro & $\begin{array}{c}\text { Intervalo de } \\
\text { Confianza }\end{array}$ & t & P-valor \\
\hline LP & $-0,13-0,20$ & 0,433 & 0,669 \\
\hline LE & $-0,02-0,18$ & 1,72 & 0,107 \\
\hline $\mathrm{L}^{*}$ & $-6,87-5,78$ & $-0,18$ & 0,859 \\
\hline $\mathrm{a}^{*}$ & $-1,32-8,94$ & 1,61 & 0,132 \\
\hline $\mathrm{b}^{*}$ & $-3,26-2,77$ & $-0,17$ & 0,863 \\
\hline
\end{tabular}

LP: longitud polar; LE: longitud ecuatorial; L*: (luminosidad) de negro; a*: cromaticidad de rojo-púrpura; b*: cromaticidad de amarillo.

Tabla 3. Morfología de 60 accesiones de semilla en Vicia faba, colectadas en Nariño, Colombia y depositadas en la colección GRISAV.

\begin{tabular}{|c|c|c|c|c|c|c|c|c|}
\hline \multirow{2}{*}{\multicolumn{2}{|c|}{ Parámetros }} & \multicolumn{7}{|c|}{ Variedades de haba } \\
\hline & & \multirow{2}{*}{$\begin{array}{l}\text { Alp } \\
3,05\end{array}$} & \multirow{2}{*}{$\begin{array}{c}\mathbf{A r} \\
2,28\end{array}$} & \multirow{2}{*}{$\begin{array}{c}\mathbf{B n} \\
2,32\end{array}$} & \multirow{2}{*}{$\begin{array}{c}\mathbf{B c} \\
2,55\end{array}$} & \multirow{2}{*}{$\begin{array}{c}\text { Ro } \\
2,51\end{array}$} & \multirow{2}{*}{\begin{tabular}{|c|} 
Rs \\
2,41
\end{tabular}} & \multirow{2}{*}{$\begin{array}{c}\mathbf{S v} \\
2,49\end{array}$} \\
\hline & $\bar{x}$ & & & & & & & \\
\hline $\mathbf{L P}$ & ds & 0,17 & 0,10 & 0,21 & 0,18 & 0,01 & 0,25 & - \\
\hline \multirow{2}{*}{ LE } & $\overline{\mathrm{x}}$ & 2,28 & 1,83 & 1,78 & 2,00 & 1,90 & 1,80 & 1,85 \\
\hline & $\mathrm{ds}$ & 0,03 & 0,10 & 0,17 & 0,15 & 0,11 & 0,19 & - \\
\hline \multirow{2}{*}{ AR } & $\bar{x}$ & 4,83 & 3,11 & 3,10 & 3,77 & 3,56 & 3,33 & 3,58 \\
\hline & ds & 0,47 & 0,31 & 0,54 & 0,51 & 0,25 & 0,63 & - \\
\hline \multirow{2}{*}{ LP/LE } & $\overline{\mathrm{x}}$ & 1,31 & 1,23 & 1,30 & 1,27 & 1,32 & 1,35 & 1,34 \\
\hline & $\mathrm{ds}$ & 0,05 & 0,05 & 0,04 & 0,07 & 0,07 & 0,05 & - \\
\hline \multirow{2}{*}{ GR } & $\bar{x}$ & 0,65 & 0,66 & 0,56 & 0,64 & 0,64 & 0,63 & 0,62 \\
\hline & $\mathrm{ds}$ & 0,14 & 0,04 & 0,06 & 0,05 & 0,04 & 0,07 & - \\
\hline \multirow{2}{*}{ P100 } & $\overline{\mathrm{x}}$ & 244,5 & 160,9 & 144,2 & 177,9 & 183,0 & 171,2 & 191,4 \\
\hline & $\mathrm{ds}$ & 12,33 & 40,47 & 18,90 & 33,87 & 27,52 & 23,51 & - \\
\hline \multicolumn{2}{|c|}{$\begin{array}{l}\text { Número de } \\
\text { accesiones }\end{array}$} & 3 & 5 & 3 & 29 & 11 & 8 & 1 \\
\hline
\end{tabular}

*LP: Longitud polar; LE: Longitud ecuatorial; AR: Área de la semilla; GR: Grosor de la semilla; P100: Peso 100 semillas; dc: desviación estándar; Alp= Alpargata; Ar= Argentina; $\mathrm{Bn}=$ Beso de novia; $\mathrm{Bc}=$ Blanca común; Ro=Roja; Rs=Rosada; Sv=Semiverde.

Estos valores son similares a los estudios realizados en México, por Pérez et al. (2015) y Salamanca et al. (2018), quienes reportan un rango de LP, entre 2,03 a 3,17 $\mathrm{cm}$ y LE desde 1,41 a 2,14 cm, pero menor al valor obtenido por De Cillis et al. (2019), quienes evaluaron seis materiales de la región de Puglia, Italia, registrando una LP, entre 2,1 a 2,3 cm y una LE, de 1,3 a 1,5 cm, posiblemente, esta diferencia se deba al hecho que estos autores evaluaron semillas de agricultor, normalmente, de menor tamaño que las variedades comerciales.

La estimación de la LP superior a 2,0 cm permitió clasificar la totalidad de la colección de $V$. faba dentro de la subespecie botánica major, cultivada para consumo humano, a nivel mundial, que se diferencia de las subespecies minor y equina, por el tamaño de la semilla (Alghamdi et al. 2017; Alharbi \& Adhikari, 2020).

Por otra parte, se destaca alta dispersión en el AR promedio de las semillas, mostrando un rango de 3,10-4,83 $\mathrm{cm}^{2}$ (Tabla 3). Para Duc et al. (2015), esta variable junto a los valores de longitud, son considerados como descriptores relevantes para la especie, lo que permite captar información de la diversidad genética entre accesiones. También, pueden ser utilizados como indicadores de selección en el mejoramiento genético, por su relación con el rendimiento en campo (Yildiz et al. 2016; Di Cecco et al. 2019; Alharbi \& Adhikari, 2020). 
A partir de los valores morfológicos, las variedades Beso de novia, Argentina y Rosada fueron catalogadas como pequeñas; las variedades Semiverde, Roja y Blanca común, se agruparon con tamaño medio y la variedad Alpargata fue definida como un material de semillas grandes (Tabla 3). Este agrupamiento propuesto para describir las variedades de haba cultivadas en Nariño, se generó a partir de los criterios cuantitativos, que concordaron con las categorías de tamaño de semilla y la asignación realizada por Sañudo et al. (1999).

En esta región, la comercialización de materiales con semilla mediana a grande y de color blanco es mayor, razón por la cual, las variedades Alpargata y Blanca común representan $80 \%$ de los cultivos de Nariño (Sañudo et al. 1999). Las variedades de haba con semillas de menor tamaño son, usualmente, empleadas como un cultivo para el autoconsumo y como una estrategia de recuperación de suelos de baja fertilidad, por la incorporación de material vegetal y el aporte de nitrógeno, cualidades también referidas en México, por Díaz et al. (2008).

Además, se pudo establecer que el tamaño de la semilla para el productor constituye un carácter importante al momento de planificar la siembra, al realizar selección de semillas grandes dentro de la variedad utilizada, con el fin de mejorar la tasa de emergencia y vigor de la planta. $\mathrm{Al}$ respecto, se ha determinado que, efectivamente, la morfología de la semilla presenta una correlación estadística significativa con las variables altura de planta, número de folíolos, número de vainas y número de tallos (Pérez et al. 2015; Alharbi \& Adhikari, 2020).

Otro rasgo abordado fue la relación entre las dos longitudes, en el índice LP/LE, que permitió aproximarse a la forma observada en las semillas. Valores menores a 1,24 fueron característicos de semillas con morfología ovada ancha, en donde el ápice inferior de la semilla fue más amplio que el superior; a medida que la relación fue mayor, las semillas presentaron una forma arriñonada, ovadareniforme (LP/LE de 1,24 a 1,32) y elipsoide-reniforme (LP/LE mayor a 1,32$)$.

A partir de los valores de referencia propuestos, $13 \%$ de los cultivares fueron clasificados en la forma ovada ancha, característico de la variedad Argentina. La forma ovada-reniforme fue la más frecuente en la colección, con el $64 \%$, distintiva en las variedades Alpargata, Beso de novia, Blanca común y Roja, igualmente, típica en materiales peruanos y mexicanos (Horque, 2004; Salamanca et al. 2018). Por el contrario, las variedades Rosada y Semiverde presentaron una característica elipsoide-reniforme, que representó el $23 \%$, que agrupa a las semillas más largas.

Al continuar con esta descripción, se observó que en las accesiones el grosor de la semilla presentó un valor semejante, con un promedio entre $0,62 \mathrm{~cm}$, en la variedad Semiverde, a 0,66 en la variedad Argentina (Tabla 3); estos valores estuvieron por debajo del rango reportado por Pérez et al. (2015) y Salamanca et al. (2018), de 0,68 a 1,40 cm y dado que no hay una descripción detallada de la metodología empleada para conocer GR por los anteriores autores, esta diferencia, se podría deber al lugar, en el cual, se realizó la medición, presumiblemente, con mayor coincidencia, si en este estudio se hubiese realizado en el extremo superior de la semilla.

Sobre esta variable no se encontró una asociación clara respecto a las variedades evaluadas. También, Salamanca et al. (2018) pudieron establecer este rasgo, al indicar que el grosor no varía proporcionalmente con el peso o tamaño de las semillas de los materiales de haba.

La variable peso de cien semillas registró valores promedios entre variedades de 144,2 - 244,5 g (Tabla 3). Que, al seguir con los planteamientos de Duc et al. (2015) y Alharbi \& Adhikari (2020), materiales de haba, con un valor mayor a $100 \mathrm{~g}$, están relacionados con el grupo taxonómico major, lo que confirma la clasificación que se había sugerido a partir de LP.

Las variedades de semillas pequeñas de forma general mostraron un P100 menor a $180 \mathrm{~g}$; las de tamaño mediano, entre 180 a $230 \mathrm{~g}$ y en el tamaño grande, un valor superior $230 \mathrm{~g}$; de estos dos últimos grupos, se destacan, por el peso, las accesiones Alp-57 (253,2 g), Alp-9 (243,6 g), Alp-5 (235,8 g), Bc-16 (226 g) y Bc-14 (221,6 g), colectados en los municipios de Pasto, Tuquerres y Carlosama.

Si se comparan estos valores con el promedio reportado por Horque (2004), de 144,7 g, Rebaa et al. (2017), con 161,0 g y Salamanca et al. (2018), con 185,5 g, es posible identificar a diferentes accesiones de la colección GRISAV, como altamente deseadas en el mercado de grano seco en esta leguminosa, por lo cual, se debería explorar el potencial de aceptación nacional y de exportación.

Color de la semilla. En apoyo a la identificación cualitativa de los productores de haba, los materiales se distribuyeron en semillas blancas (59 \%), rojas $(17 \%)$, rosadas $(13 \%)$ y verdes $(3 \%)$. Además, la colección contó con tres accesiones de la variedad Beso de novia $(8 \%)$, con presencia de color secundario.

En la tabla 4, se puede visualizar representantes de las variedades de Vicia faba estudiadas y el valor promedio de los parámetros CIELab. La clasificación, a partir del color de la semilla, coincide con varios registros de la especie, además, concuerda con un mayor reporte de frecuencia de color blanco o crema (Duc et al. 2015; Karkanis et al. 2018; De Cillis et al. 2019); sin embargo, no se identificaron materiales negros, grises o amarillos, también referidos por Horque (2004) y Karkanis et al. (2018).

Es necesario indicar que el color está relacionado con el cambio de las semillas de verde a otras tonalidades, ya que la clorofila se descompone y se acumulan nuevos compuestos durante la maduración (De Cillis et al. 2019), por lo cual, la diversidad presentada en el haba, posiblemente, se deba a mutaciones específicas en algunos genes, que se estabilizan en las poblaciones y han permitido realizar selección y clasificación, tanto a productores como a mejoradores (Yildiz et al. 2016). 
Tabla 4. Estimación de parámetros CIELab de semilla de Vicia faba, colectadas en Nariño, Colombia y depositadas en la colección GRISAV.

\begin{tabular}{|c|c|c|c|c|c|}
\hline Variedad* & CIELab & dst. & Variedad* & CIELab & dst. \\
\hline & $\begin{array}{l}\text { L: } 50,53 \\
a^{*}: 3,69 \\
\text { b*: } 32,17\end{array}$ & $\begin{array}{l}\mathbf{L}: \pm 4,8 \\
\mathbf{a}^{*}: \pm 0,1 \\
\text { b*: } \pm 4,4\end{array}$ & & $\begin{array}{l}\text { L: } 57,28 \\
\text { a*: }-6,24 \\
\text { b*: } 32,13\end{array}$ & $\begin{array}{l}\text { L: } \pm 6,9 \\
\text { a*: } \pm 6,0 \\
\text { b*: } \pm 2,7\end{array}$ \\
\hline & $\begin{array}{l}\text { L: } 60,95 \\
\text { a*: }^{*}-4,79 \\
\text { b*: } 31,24\end{array}$ & $\begin{array}{l}\mathbf{L}: \pm 5,3 \\
\mathbf{a}^{*}: \pm 3,9 \\
\mathbf{b}^{*}: \pm 2,3\end{array}$ & & $\begin{array}{l}\text { L: } 16,43 \\
\text { a*: } 13,48 \\
\text { b*: } 5,09\end{array}$ & $\begin{array}{l}\text { L: } \pm 4,3 \\
\text { a*: } \pm 3,3 \\
\text { b*: } \pm 2,5\end{array}$ \\
\hline & $\begin{array}{l}\mathbf{L}_{1}: 59,07 \\
\mathbf{a}_{\mathbf{1}} \boldsymbol{*}:-6,98 \\
\mathbf{b}_{1} *: 32,77\end{array}$ & $\begin{array}{l}\mathbf{L}: \pm 8,3 \\
\mathbf{a}^{*}: \pm 3,9 \\
\mathbf{b} * \pm 0,9\end{array}$ & & $\begin{array}{l}\text { L: } 31,90 \\
\text { a*: } 15,44 \\
\text { b*: } 17,60\end{array}$ & $\begin{array}{l}\mathbf{L}: \pm 2,9 \\
\text { a*: } \pm 5,3 \\
\text { b*: } \pm 1,2\end{array}$ \\
\hline & $\begin{array}{l}\mathbf{L}_{2}: 12,11 \\
\mathbf{a}_{2} *: \\
\mathbf{b}_{2} *: \\
\mathbf{b}^{*}, 58\end{array}$ & $\begin{array}{l}\mathbf{L}_{2}: \pm 8,6 \\
\mathbf{a}_{2} *: \pm 4,2 \\
\mathbf{b}_{2} *: \pm 6,1\end{array}$ & & $\begin{array}{l}\text { L: } 49,77 \\
\mathbf{a}^{*}:-12,45 \\
\text { b*: } 29,04^{*}\end{array}$ & $\begin{array}{l}\text { L: }+2,0 \\
\mathbf{a}^{*}: \pm 0,9 \\
\text { b*: }^{*} \pm 1,7\end{array}$ \\
\hline
\end{tabular}

*Alp= Alpargata; $\mathrm{Ar}=$ Argentina; $\mathrm{Bn}=$ Beso de novia; $\mathrm{Bc}=\mathrm{Blanca}$ común; Ro=Roja; Rs=Rosada; $\mathrm{Sv}=\mathrm{Semiverde}$.

La cuantificación de esta característica, resumida en la tabla 4, puede reducir la subjetividad vinculada con la inspección visual, dotando de valores numéricos para la comparación del germoplasma entre variedades y accesiones, respaldado, además, en el hecho, que no se encontraron diferencias estadísticas, mediante la prueba de $\mathrm{T}$ Student, entre el método digital y el espectrofotómetro, presentando una variación inferior a $17 \%$, que es asumido como aceptable, por Varga et al. (2019), al comparar los mismos métodos en semillas de fríjol (Phaseolus vulgaris L.).

El grupo de semillas de las variedades Argentina, Alpargata, Blanca común y Beso de novia (color principal) mostraron un valor de luminosidad $\left(\mathrm{L}^{*}\right)$ por encima de 50 , describiendo una tonalidad clara que, junto a valores bajos y negativos en el eje $a^{*}$, con valores altos y positivos en el eje $\mathrm{b}^{*}$, alude una combinación que tiende al amarillo (Tabla 4).

Khazaei \& Vandenberg (2020) encontraron que los colores claros en estas semillas, se relacionan con mayores contenidos de proteína, en comparación con semillas de otro color; además, estos autores registraron proporciones importantes de $\mathrm{Ca}, \mathrm{Mg}, \mathrm{Fe}$ y $\mathrm{Zn}$, en las semillas claras, que son minerales que, a menudo, faltan en la dieta humana.

Por el contrario, las semillas rosadas y rojas están asociadas con la presencia de compuestos flavonoides, antocianinas y taninos (Yildiz et al. 2016; Khazaei \& Vandenberg, 2020). En este trabajo, los parámetros CIELab presentaron un valor positivo y alto en el eje $\mathrm{a}^{*}$, que indica mayor influencia del color rojo; un valor bajo en el eje ortogonal $b^{*}$ y la luminosidad fue distintiva, siendo mayor en la variedad Rosada y menor en la variedad Roja (Tabla 4).

La variedad identificada como Semiverde presentó el valor negativo más alto en el parámetro $\mathrm{a}^{*}$ (tonalidad verde), junto a valores de $\mathrm{L}^{*}$ y $\mathrm{b}^{*}$, que indican alta luminosidad y tonalidad secundaria amarilla (Tabla 4); posiblemente, estas semillas presenten concentraciones residuales de clorofilas a y b, como lo ha mencionado De Cillis et al. (2019).

Finalmente, relacionado al color, se identificó que solo los materiales Bc-55 y Rs-58 presentaron semillas con hilio claro en la colección, una característica poco común, debido al predominio del color negro. Respecto a esto, Duc et al. (2015) determinaron que esta condición involucra a los genes recesivos zt-1 y zt-2, que controlan la ausencia de taninos en esta estructura. Al ser una característica poco estudiada, no se encontraron reportes sobre relaciones funcionales o diferencias productivas, por lo cual, sería importante estudiar esta condición, en trabajos futuros.

Análisis de correlación. A partir de la tabla 5, se destaca la relación que se presentó entre la longitud polar y ecuatorial con las demás variables evaluadas, lo que confirma la importancia de estas mediciones, al estudiar la morfología de las semillas de haba (Duc et al. 2015; De Cillis et al. 2019). 
Tabla 5. Análisis de correlación de variables morfológicas y de color en semillas de Vicia faba, colectadas en Nariño, Colombia y depositadas en la colección GRISAV.

\begin{tabular}{|l|c|c|c|c|c|c|c|c|c|}
\hline & LP & LE & LP/LE & AR & GR & P100 & L & a & b \\
\hline LP & 1 & $* *$ & $* *$ & $* *$ & ns & $* *$ & ns & ns & ns \\
\hline LE & 0,69 & 1 & $* *$ & $* *$ & ns & $* *$ & $* *$ & $* *$ & $* *$ \\
\hline LP/LE & 0,25 & $-0,53$ & 1 & ns & ns & ns & $* *$ & $* *$ & $* *$ \\
\hline AR & 0,94 & 0,86 & $-0,05$ & 1 & ns & $* *$ & $*$ & $* *$ & $* *$ \\
\hline GR & 0,02 & 0,08 & $-0,08$ & 0,05 & 1 & $*$ & $\mathrm{~ns}$ & $\mathrm{~ns}$ & $\mathrm{~ns}$ \\
\hline P100 & 0,63 & 0,60 & $-0,04$ & 0,67 & 0,12 & 1 & $\mathrm{~ns}$ & $\mathrm{~ns}$ & $\mathrm{~ns}$ \\
\hline $\mathbf{L}$ & 0,05 & 0,18 & $-0,20$ & 0,12 & $-0,01$ & $-0,09$ & 1 & $* *$ & $* *$ \\
\hline $\mathbf{a}$ & $-0,09$ & $-0,26$ & 0,18 & $-0,16$ & $-0,03$ & 0,05 & $-0,83$ & 1 & $* *$ \\
\hline $\mathbf{b}$ & 0,10 & 0,26 & $-0,18$ & 0,17 & 0,00 & $-0,02$ & 0,90 & $-0,76$ & 1 \\
\hline
\end{tabular}

*LP: Longitud polar; LE: Longitud ecuatorial; AR: Área de la semilla; GR: Grosor de la semilla; P100: Peso 100 semillas; L: luminosidad de color; a: posición rojo-verde; b: posición amarillo-azul; *: P-valor <0,05; **: P-valor: <0,01; ns: no significancia.

Valores altos de LP y LE mostraron una correspondencia fuerte y positiva con el área y el peso del grano $(r>0,6)$ que, a su vez, se encuentran asociadas significativamente (AR x P100: $r=0,67$ ). Este comportamiento fue similar al evidenciado por Pérez et al. (2015) y Salamanca et al. (2018), quienes concluyeron que, en esta especie, las semillas de mayor tamaño presentan también un mayor peso. Además, LE evidenció una correlación baja con los parámetros de color $\mathrm{L}^{*} \mathrm{y} \mathrm{b}^{*}$, lo que indica que las variedades de tonalidad clara presentaron semillas con longitud ecuatorial mayor $(r=0,18$ y 0,26$)$, a diferencia de las semillas con tendencia al color rojo $\left(a^{*}\right)$, las cuales, mostraron una menor longitud $(r=-0,26)$, comportamiento que está asociado con las formas encontradas.

Por último, la correlación entre $L^{*}, a^{*}$ y b* confirma que estos parámetros son dependientes entre sí y aportan cada uno una parte sustancial de información del color de la semilla (Varga et al. 2019). $\mathrm{L}^{*} \times \mathrm{b}^{*}(r=0,90)$ presentó una correlación alta positiva a diferencia de las variables $L^{*} \mathrm{x} \mathrm{a}^{*} \mathrm{y} \mathrm{a}^{*} \mathrm{x} \mathrm{b}^{*}$, con un coeficiente de Pearson de $r=-0,83$ y $-0,76$, que muestra la influencia de la saturación de los colores sobre la luminosidad y la presencia de tonalidades marcadas dentro de la colección, con color blanco, rojo, rosado y verde.
Análisis de componentes principales (ACP). Este método multivariado, se utiliza para obtener una perspectiva de la variabilidad de las semillas en diferentes especies de leguminosas. Cuanto mayor es la dispersión de los materiales dentro de los cuadrantes del biplot, se incrementa la variabilidad, la cual, decrece al presentar aglomeraciones entre individuos, que pueden reflejar una base genética más estrecha (Rebaa et al. 2017; De Cillis et al. 2019).

En este estudio, fueron seleccionados los tres primeros componentes al presentar un valor propio mayor a 1,0 y explicar una variación total del 93,1\%. Las variables LP, LE, AR y P100, asociadas con la forma y peso de la semilla, fueron los principales contribuyentes del CP1 (41,6\%); los parámetros de color L*, a* y b* fueron representados en CP2 $(30,2 \%)$ y las variables morfológicas LP/LE y GR, contribuyeron a la conformación de CP3 (21,3\%).

El biplot generado (Figura 1) muestra una nube de puntos dispersos, que permitió identificar, en el lado negativo del eje 1 , las accesiones con mayor tamaño, entre estas, se destacan Alp-57, Alp-5, Bc-14, Bc20 y Alp-9, que representan uno de los grupos propuestos. En el

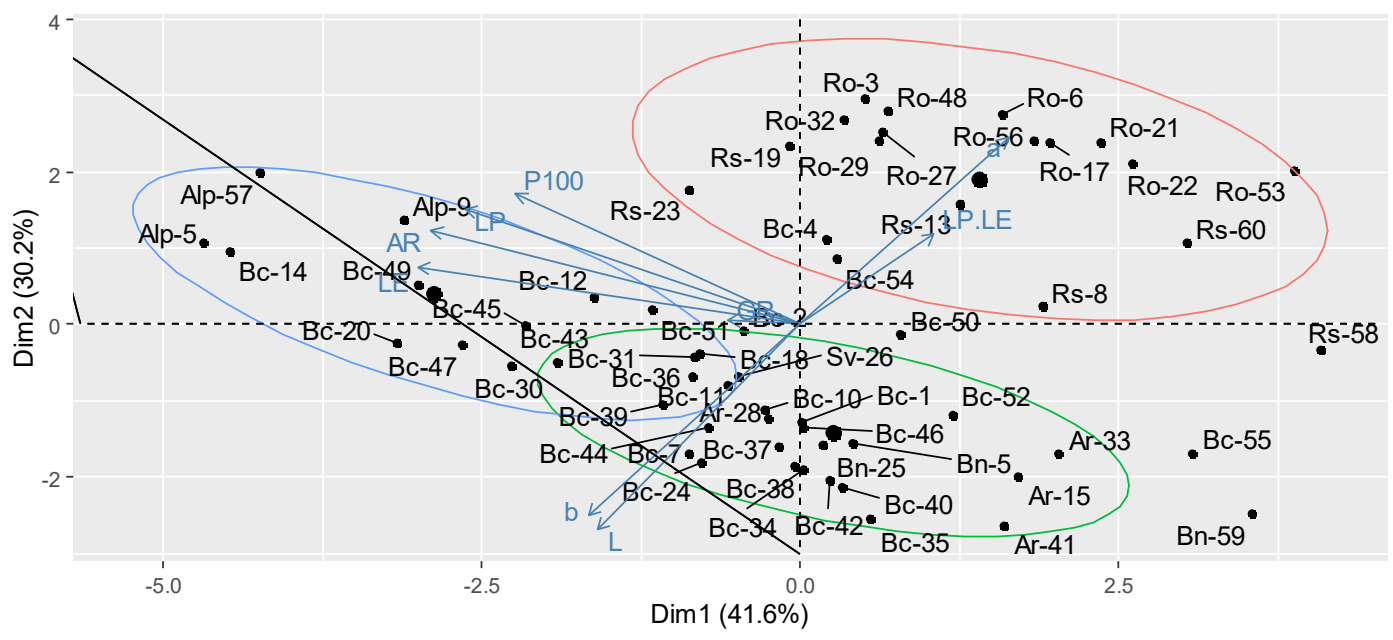

Figura 1. Biplot de variables y accesiones de haba de la colección GRISAV, en el plano factorial de los ejes 1 y 2*. *Alp= Alpargata; $\mathrm{Ar}=$ Argentina; $\mathrm{Bn}=$ Beso de novia; $\mathrm{Bc}=\mathrm{Blanca}$ común; Ro=Roja; Rs=Rosada; $\mathrm{Sv}=$ Semiverde. 
cuadrante ortogonal, se situó un segundo grupo de accesiones con características inversas, donde sobresalen materiales, como Bn-59, Bc-55 y Ar-41, Ar-33 y Ar-15.

Un tercer grupo presentó características intermedias respecto a la morfología de la semilla y, particularmente, una forma más alargada por la relación LP/LE; además, éste fue separado a partir del color, ubicándose en referencia al eje 2, en el cuadrante positivo. Todos los materiales de la variedad Roja y Rosada integraron este tercer grupo, opuesto a las semillas claras, que se ubicaron en el cuadrante negativo, del mismo eje (Figura 1).

A propósito, Varga et al. (2019) identificaron en semillas de frijol, que los parámetros de color, cuando son integrados al análisis de diversidad, aportan una cantidad indispensable de información en el ACP y contribuyen, de forma significativa, a la conformación de grupos, dentro de una colección.

En trabajos similares, se explica que, parte de la diferenciación morfológica y de color de las semillas de haba, se dio como resultado de la selección dirigida a favorecer características de interés y del intercambio por parte de los productores entre regiones (Herrera et al. 2013; Rebaa et al. 2017; Karkanis et al. 2018), una práctica común, a partir del "trueque" o cambio y del préstamo de semilla, entre agricultores en Nariño.

El ambiente, también es considerado como un factor que puede influir sobre el genotipo y el fenotipo de los materiales de haba, lo que provoca respuestas cuantificables sobre la morfología de las semillas (Salamanca et al. 2018; Alharbi \& Adhikari, 2020), es decir, en ambiente favorable, el tamaño de las semillas aumenta y en ambientes restrictivos, el tamaño disminuye, por lo que se ha propuesto una alta plasticidad fenotípica en las especies leguminosas. Incluso, Di Cecco et al. (2019) establecieron la relación entre la precipitación de una región y cambios en la tonalidad del color de las semillas de Astragalus aquilanus.

Basados en la tasa de polinización cruzada natural del haba, que oscila entre 10 y $70 \%$, se debe considerar el flujo genético como un factor de variabilidad en el genotipo y el fenotipo (Duc et al. 2015; Alghamdi et al. 2017), lo que indica que, bajo situaciones de polinización cruzada, el color de las semillas podría cambiar de una generación a otra. Al respecto, Rebaa et al. (2017) encontraron, en estudios realizados en Tunes, elevado flujo de genes entre localidades geográficas, debido a la acción de los insectos polinizadores, como abejorros y una gran diversidad de abejas solitarias, de los géneros Anthopora y Xylocarpa.

Lo anterior indica, que evaluar la semilla de haba para conocer su diversidad a partir de la forma y color de la semilla debe, ser acompañada de evaluaciones complementarias, porque puede no ser repetible, por el efecto del ambiente, del cruzamiento y de la interacción genotipo por ambiente; sin embargo, le da a la especie varias estrategias para adaptarse al entorno ecológico, en donde la producción de semillas heterogéneas, probablemente, sea una consecuencia de ello y que permite confirmar que en Nariño existe diversidad asociada al cultivo, un recurso fundamental para establecer y gestionar acciones de conservación, responder a las necesidades de los productores y proporcionar fuentes de germoplasma, útiles para los mejoradores.

Conflicto de intereses: El manuscrito fue preparado y revisado con la participación de todos los autores, quienes declaramos que no existe ningún conflicto de intereses que ponga en riesgo la validez de los resultados presentados. Financiación: Esta investigación fue financiada por la Gobernación de Nariño y CEIBA, proyecto "Fortalecimiento de Capacidades Regionales en Investigación, Desarrollo Tecnológico e Innovación en el Departamento de Nariño".

\section{REFERENCIAS}

1. ALGHAMDI, S.; AL-FAIFI, S.; MIGDADI, H.; AL-ROWAILY, S.; EL-HARTY, E.; FAROOQ, M. 2017. Genetic diversity and field performance of mung bean, faba bean and lentil genotypes in the Kingdom of Saudi Arabia. Internal J. Agriculture and Biology (Pakistán). 19(4):689-696. https://doi.org/10.17957/IJAB/15.0336

2. ALHARBI, N.; ADHIKARI, K. 2020. Factors of yield determination in faba bean (Vicia faba). Crop and Pasture Science (Australia). 71(4):305-321. https://doi.org/10.1071/CP19103

3. ÁlVAREZ, D.; GÓMEZ, E.; ORDÓÑEZ, H. 2019. Tipología de fincas productoras de arveja (Pisum sativum L.) en la subregión Sur de Nariño, Colombia. Cienc Tecnol Agropecuaria (Colombia). 20(3):659-677. https://doi.org/10.21930/rcta.vol20_num3_art:1593

4. DE CILLIS, F.; LEONI, B.; MASSARO, M.; RENNA, M.; SANTAMARIA, P. 2019. Yield and quality of faba bean (Vicia faba L. var. major) genotypes as a vegetable for fresh consumption: A comparison between Italian landraces and commercial varieties. Agriculture (Suiza). 9:253-267. https://doi.org/10.3390/agriculture9120253

5. DE LA ROSA, L.; FAJARDO, J. 2016. La agrobiodiversidad como elemento de la seguridad alimentaria y ambiental. Arbor (España). 192(779):a316. http://dx.doi.org/10.3989/arbor.2016.779n3006

6. DI CECCO, V.; DI MUSCIANO, M.; D'ARCHIVIO, A.; FRATTAROLI, A.; DI MARTINO, L. 2019. Analysis of intraspecific seed diversity in Astragalus aquilanus (Fabaceae), an endemic species of Central Apennine. Plant Biology (Holanda). 21(3):507-514. https://doi.org/10.1111/plb.12844

7. DÍAZ, M.; HERRERA, B.; RAMÍREZ, J.; ALIPHAT, M.; DELGADO, A. 2008. Conocimiento campesino en la selección de variedades de haba (Vicia faba L.) en la 
sierra norte de Puebla México. Interciencia (México). 33(8):610-615.

8. DUC, G.; ALEKSIC, J.; MARGET, P.; MIKIC, A.; PAULL, J.; REDDEN, R.; TORRES, A. 2015. Faba bean. En: De Ron, A. (Ed.). Grain legumes. Ed. Springer (UK). p.141-178.

9. HERRERA, B.; CASTILLO, F.; ORTEGA, R.; DELGADO, A. 2013. Poblaciones superiores de la diversidad de maíz en la región oriental del estado de México. Revista Fitotecnia Mexicana (México). 36(1):33-43.

https://doi.org/10.35196/rfm.2013.1.33

10. HORQUE, R. 2004. Cultivo del haba. Ed. INIA (Perú). 52p.

11. KARKANIS, A.; NTATSI, G.; LEPSE, L.; FERNÁNDEZ, J.; VÅGEN, I.; REWALD, B.; SAVVAS, D. 2018. Faba bean cultivation: revealing novel managing practices for more sustainable and competitive European cropping systems. Frontiers in Plant Science (Suiza). 9:1-14. https://doi.org/10.3389/fpls.2018.01115

12. KHAZAEI, H.; VANDENBERG, A. 2020. Seed mineral composition and protein content of faba beans (Vicia faba L.) with contrasting tannin contents. Agronomy (Suiza). 10(511):2-10.

https://doi.org/10.3390/agronomy10040511

13. MINISTERIO DE AGRICULTURA Y DESARROLLO RURAL, MADR. 2021. Área, producción, rendimiento y participación municipal de haba.

Disponible desde Internet en:

https://n9.cl/jOpav (con acceso 4/10/2021).

14. PÉREZ, D.; GONZÁlEZ, A.; RUBÍ, M.; MORA, F.; PASCUAL, J.; PADILLA, A. 2015. Análisis de 35 cultivares de haba por su producción de vaina verde y otros componentes del rendimiento. Rev. Mex. Ciencias Agrícolas (México). 6(7):1601-1613.

https://doi.org/10.29312/remexca.v6i7.553

15. REBAA, F.; ABID, G.; AOUIDA, M.; ABDELKARIM, S.; AROUA, I.; MUHOVSKI, Y.; JEBARA, M. 2017. Genetic variability in Tunisian populations of faba bean (Vicia faba L. var. major) assessed by morphological and SSR markers. Physiology and Molecular Biology of Plants (India). 23(2):397-409.

https://doi.org/10.1007/s12298-017-0419-x

16. SALAMANCA, G.; DELGADO, A.; HERRERA, B.; MENDOZA, M.; CONDE, V. 2018. Variación en tamaño de grano y rendimiento de almidón en cultivares de Vicia faba L. Agro Productividad (México). 11(7):67-72.

17. SAÑUDO, B.; CHECA, O.; ARTEAGA, G. 1999. Manejo agronómico de leguminosas en zonas cerealistas de Nariño. Ed. Universidad de Nariño (Colombia). 59p.

18. VARGA, F.; VIDAK, M.; IVANOVIĆ, K.; LAZAREVIĆ, B.; ŠIRIĆ, I.; SREČEC, S.; CAROVIĆ-STANKO, K. 2019. How does computer vision compare to standard colorimeter in assessing the seed coat color of common bean (Phaseolus vulgaris L.)? J. Central European Agriculture (U.E.). 20(4):1169-1178.

http://dx.doi.org/10.5513/JCEA01/20.4.2509

19. YILDIZ, G.; CIL, A.; TIRYAKI, I. 2016. Revealing seed coat colour variation and their possible association with seed yield parameters in common vetch (Vicia sativa L.). International Journal of Agronomy (E.E.U.U.). 2016:1-10. http://dx.doi.org/10.1155/2016/1804108 